\title{
MicroRNA-1254 exerts oncogenic effects by directly targeting RASSF9 in human breast cancer
}

\author{
BIN LI ${ }^{1,2}$, PENG CHEN $^{3}$, JIALIN WANG ${ }^{2}$, LINXIA WANG $^{4}$, MINGZHI REN ${ }^{2}$, \\ RUISAN ZHANG ${ }^{3}$ and JIANJUN HE ${ }^{1}$ \\ ${ }^{1}$ Department of Breast Surgery, The First Affiliated Hospital of Xi'an Jiaotong University, Xi'an, \\ Shaanxi 710061; ${ }^{2}$ Affiliated Baoji Hospital of Xi'an Medical University, Baoji, Shaanxi 721006; \\ ${ }^{3}$ Institution of Basic Medical Science, Xi'an Medical University, Xi'an, Shaanxi 710021; \\ ${ }^{4}$ Foreign Language College, Xi'an Technological University, Xi'an, Shaanxi 710059, P.R. China
}

Received January 29, 2018; Accepted July 30, 2018

DOI: $10.3892 /$ ijo.2018.4530

\begin{abstract}
MicroRNAs (miRNAs or miRs) play crucial roles in human breast cancer. Although miR-1254 has been shown to have oncogenic activity in several cancer types, its biological function in breast cancer and its mechanisms of action remain unclear. In this study, we investigated the role of miR-1254 in human breast cancer and sought to elucidate the relevant underlying mechanisms. We found that miR-1254 expression was markedly increased in breast cancer tissues and cell lines. Additionally, miR-1254 overexpression accelerated breast cancer cell proliferation, cell cycle G1-S phase transition and inhibited apoptosis. Nevertheless, the inhibition of miR-1254 suppressed cell proliferation and induced apoptosis. Further analyses revealed that miR-1254 expression negatively correlated with RASSF9 expression in breast cancer tissues. We verified that RASSF9 was a direct target of miR-1254 using a luciferase reporter assay. The overexpression of miR-1254 reduced the RASSF9 mRNA and protein levels, and the suppression of miR-1254 promoted RASSF9 expression. Notably, the knockdown or overexpression of RASSF9 corroborated the biological effects observed upon miR-1254 overexpression or inhibition. Taken together, these results demonstrate that miR-1254 accelerates breast cancer cell growth by activating the AKT signaling pathway and suppresses apoptosis by inhibiting p53 expression through the targeting of RASSF9. The data indicate that miR-1254 plays a crucial role in human breast cancer, and may represent a novel therapeutic target for this malignancy.
\end{abstract}

Correspondence to: Professor Jianjun He or Dr Bin Li, Department of Breast Surgery, The First Affiliated Hospital of Xi'an Jiaotong University, 277 West Yanta Road, Xi'an, Shaanxi 710061, P.R. China E-mail:49538373@qq.com

E-mail: libinxyfy@163.com

Key words: breast cancer, microRNA-1254, Ras association domain-containing protein 9 , proliferation, apoptosis

\section{Introduction}

Human breast cancer is one of the most commonly diagnosed cancers worldwide (1). It is estimated that the number of patients with breast cancer increases annually by $\sim 1.7$ million globally; $\sim 245,000$ new cases of breast cancer and $>45,000$-related are recorded in the USA each year, as well as $\sim 170,000$ new cases and $>40,000$-related deaths in China $(2,3)$. Although breast cancer diagnosis and therapy have both improved, satisfactory therapeutic effects have not yet been achieved due to disease complexity. Tumorigenesis is a multifactorial and multistep process involving a range of genetic alterations, including the activation of oncogenes, the inactivation of anti-oncogenes and the abnormal expression of cancer-related genes (4-6). Previous studies have unraveled some of the pathological mechanisms involved in breast tumorigenesis $(7,8)$; however, these have yet to be fully elucidated. Hence, it is important to fully characterize the molecular mechanisms responsible for breast cancer progression, and these may then be used to identify novel biomarkers and therapeutic targets for early diagnosis and therapy.

MicroRNAs (miRNAs or miRs) are an abundant class of non-coding RNAs, approximately 18-24 nucleotides in length, that bind to the 3'-untranslated regions (UTRs) of target genes and regulate expression post-transcriptionally (9-11). Over the past decade, miRNAs have been demonstrated to actively participate in various biological effects, including cancer cell survival, growth, motility, autophagy and apoptosis (12-15). Numerous miRNAs reportedly function either as oncogenes or anti-oncogenes. Studies have indicated that miRNAs are often aberrantly regulated and play prohibitive or oncogenic roles in breast cancer $(16,17)$. Recently, the clinical significance of miR-1254 has been verified, along with its role in tumorigenesis and progression, including lung cancer, thyroid cancer and oral squamous cell carcinoma (18-20).

In this study, using bioinformatics software, we predicted that miR-1254 could target Ras-association domain family 9 (RASSF9). RASSF9 is a RAS-association domain family gene and is expressed in multiple tissues. The RASSF family includes 10 genes from RASSF1 to RASSF10 and is subdivided into C-terminally (RASSF1-6) and N-terminally 
(RASSF7-10). The N-terminal RASSF genes are involved in cell growth, survival and apoptosis, among other processes (21). Evidence suggests that RASSF7 promotes lung cancer cell growth, migration and invasion by inhibiting the phosphorylation of mammalian Ste20-like kinase 1, large tumor suppressor kinase 1 and yes-associated protein (22). RASSF8 suppresses cell proliferation, migration and invasion in lung cancer, gastric cancer and cervical cancer (23-26). RASSF10 acts as a novel tumor suppressor in liver cancer, breast cancer, thyroid cancer, gastric cancer, lung cancer, colorectal cancer, esophageal squamous cell carcinoma and hepatocarcinoma (27-32). However, the functions of RASSF9 in many types of cancer, including breast cancer, remain unknown.

In the present study, we investigated the function and mechanisms of action of miR-1254 in human breast cancer. We demonstrate that the expression of miR-1254 is markedly upregulated in human breast cancer tissues and is associated with certain clinicopathological characteristics. Furthermore, miR-1254 potently promotes breast cancer cell growth and cycle transition. In particular, we demonstrate that RASSF9 is a direct target of miR-1254. Thus, these findings indicate that miR-1254 may be a new target for breast carcinoma therapy.

\section{Materials and methods}

Preparation of tissue samples. Human breast cancer samples $(\mathrm{n}=79)$ were collected from patients who were diagnosed at the Department of Oncology Surgery (the First Affiliated Hospital, Xi'an Jiaotong University, Xi'an, China) between January, 2015 and March, 2016. Informed consent was obtained from each patient prior to specimen collection, and the tissues were stored at $-80^{\circ} \mathrm{C}$. Clinicopathological data were obtained by reviewing the pathological records. The study protocol was approved by the Ethics Committee of Xi'an Jiaotong University. The Cancer Genome Atlas (TCGA) datasets from 114 normal samples and 1,099 cancer samples were analyzed for miR-1254 and RASSF9 expression levels.

Cell culture. The human breast carcinoma cell lines, T47D, MCF-7, ZR-75-30 and MDA-MB-231, and the normal breast epithelial cell line, HB2, were obtained from the Cell Bank of Genechem (Genechem Co., Ltd., Shanghai, China). The cells were tested and authenticated by the Cell Bank of Genechem. The cells $\left(1 \times 10^{5}\right.$ cells $\left./ \mathrm{ml}\right)$ were cultivated in Dulbecco's modified Eagle's medium supplemented with $10 \%$ fetal bovine serum (both from Thermo Fisher Scientific, Inc., Waltham, MA, USA) and were cultured at $37^{\circ} \mathrm{C}$.

Reverse transcription-quantitative polymerase chain reaction $(R T-q P C R)$. RNA was extracted from the human tissues and breast cancer cell lines using TRIzol reagent (Invitrogen/Thermo Fisher Scientific, Inc. The SYBR Premix Ex Taq II and PrimeScript RT Reagent kits (Takara Biotechnology, Inc., Dalian, China) were used to examine miR-1254 and RASSF9 mRNA expression. RT-qPCR were performed with the iCycler iQ Multicolor RT-qPCR Detection System (Bio-Rad Laboratories, Inc., Hercules, CA, USA) under the following conditions: $95^{\circ} \mathrm{C}$ for $10 \mathrm{~min}$, followed by 45 cycles at $95^{\circ} \mathrm{C}$ for $10 \mathrm{sec}, 60^{\circ} \mathrm{C}$ for $20 \mathrm{sec}$, and $72^{\circ} \mathrm{C}$ for $30 \mathrm{sec}$. All the primer sequences were as follows: miR-1254 reverse-transcribed primer, 5'-GTCGTATCCAGTGCGTGTCGTGGAGTCGG CAATTGCACTGGATACGACCTGCAGT-3'; miR-1254 forward, 5'-ATCCAGTGCGTGTCGTG-3' and reverse, 5'-TGC TAGCCTGGAAGCTGGAGC-3'; U6 reverse-transcribed primer, 5'-CGCTTCACGAATTTGCGTGTCAT-3'; U6 forward, 5'-GCTTCGGCAGCACATATACTAAAAT-3' and reverse, 5'-CGCTTCACGAATTTGCGTGT CAT-3'; RASSF9 forward, 5'-ACAACAATCCCGCAGTTCAAA-3' and reverse, 5'-GTGTCTGGATTTCCAGGGTGA-3'; $\beta$-actin forward, 5'-TGGCACCCAGCACAATGAA-3' and reverse, 5'-CTA AGTCATAGTCCGCCTAGAAGCA-3'. The data were normalized to RNU6B (U6) or $\beta$-actin gene expression. The relative expression of genes was calculated with the $2^{-\Delta \Delta \mathrm{Cq}}$ method (33).

Expression vector construction. hsa-miR-1254 precursor expression vector (named miR-1254) and a control vector (named Control) were constructed using chemosynthetic oligonucleotides and interpolated into pcDNA6.2-GW/EmGFPmiR plasmids (both from Genechem Co., Ltd.). RASSF9 gene DNA was inserted in the pCMV2-GV146 vector (Genechem Co., Ltd.). Transfection was conducted using Lipofectamine 2000 (Invitrogen/Thermo Fisher Scientific, Inc.). Cells were transfected and cultured for $48 \mathrm{~h}$ before performing the assays.

Dual-luciferase assay. The possible target genes of miR-1254 were predicted by using bioinformatics software miRanda (www.ma.uni-heidelberg.de/apps/zmf/mirwalk/) and RegRNA (http://regrna.mbc.nctu.edu.tw/html/prediction.html). The binding site of miR-1254 in the 3'-UTR of RASSF9 was constructed and inserted in the pmirGLO Dual-Luciferase expression vector (named RASSF9-WT; Genechem Co., Ltd.). Mutated sequences of RASSF9 were also constructed (named RASSF9-MT). miR-1254 plasmids and reporter plasmids were co-transfected into 293 cells (Genechem Co., Ltd.) Cells were collected at $24 \mathrm{~h}$ post-transfection, and the reporter activity was detected using a Dual-Luciferase assay system (Promega Corp., Madison, WI, USA).

Anti-miR-1254/RASSF9 siRNA synthesis and transfection. Interfering RNA oligonucleotides served as miR-1254 inhibitor (named anti-miR-1254) and were synthesized by GenePharma (Shanghai, China). The sequence of anti-miR-1254 was 5'-ACUGCAGGCUCCAGCUUCCAGGCU-3'. Scrambled siRNA served as the control (named anti-miR-Control), and the sequence was 5'-CAGUACUUUUGUGUAGUACAA-3'. RNA oligonucleotides were transfected into breast cancer MCF-7/T47D cells using Lipofectamine 2000. Small interfering RNA (siRNA) were used to silence the human RASSF9 gene. Human RASSF9 siRNA (sense, 5'-GAGAAUGAAAGAGCU GGAUTT-3' and antisense, 5'-AUCCAGCUCUUUCAUUCU CTT-3'), and negative siRNA (NC-siRNA sense, 5'-UUCUCCG AACGUGUCACGUTT-3' and antisense, 5'-ACGUGACACG UUCGGAGAATT-3') were synthesized by GenePharma. The siRNAs were transfected into the cells using Lipofectamine 2000 and diluted to $70 \mathrm{nM}$ for use in the experiment.

MTT assay. MCF-7/T47D cells $(4,000$ cells/well in $100 \mu \mathrm{l}$ DMEM) were seeded into 96-well plates and cultivated for $24 \mathrm{~h}$, followed by transfection with the Control, miR-1254, anti-miR-Control, anti-miR-1254, NC-siRNA (70 nM), 
RASSF9 siRNA (70 nM), Vector control or RASSF9 expression vector for 24,48 or $72 \mathrm{~h}$. Cell viability was then examined by MTT assay and the absorbance was read at $492 \mathrm{~nm}$ using a microplate reader (FLUOstar OPTIMA; BMG Labtech, Ortenberg, Germany).

Cell counting assay. To measure cell growth, $1 \times 10^{5}$ cells were seeded into $60-\mathrm{mm}$-diameter plates and cultured for $24 \mathrm{~h}$, followed by transfection with the Control, miR-1254, anti-miR-Control, anti-miR-1254, NC-siRNA (70 nM), RASSF9 siRNA (70 $\mathrm{nM}$ ), Vector control or RASSF9 expression vector. Cell numbers were counted at 24,48 and $72 \mathrm{~h}$ following treatment using a Countess automated cell counter (Invitrogen, Eugene, OR, USA).

Cell cycle analysis. The MCF-7/T47D cells were incubated at $37^{\circ} \mathrm{C}$ for $48 \mathrm{~h}$, prior to harvesting and fixing in $70 \%$ ice-cold ethanol. The cells were then dyed using propidium iodide and RNase A (DNAse-free) (Sigma, St. Louis, MO, USA). Finally, the cell cycle distribution was assessed via fluorescenceactivated cell sorting (BD Biosciences, San Jose, CA, USA), and the proportions calculated with ModFit software (Bio-Rad Laboratories, Hercules, CA, USA).

Cell apoptosis analysis. The MCF-7/T47D cells were seeded into 6-well plates in triplicate and treated for $48 \mathrm{~h}$ with the Control, miR-1254, anti-miR-Control, anti-miR-1254, NC-siRNA (70 nM), RASSF9 siRNA (70 nM), Vector control or RASSF9 expression vector. An Annexin V FITC Apoptosis Detection kit (Invitrogen/Thermo Fisher Scientific, Inc.) was used to measure cell apoptosis. During the early stage of apoptosis, cells begin to display phosphatidylserine (PS) on the cell surface membranes where it is readily detectable by staining the cells with Annexin V-FITC. As the plasma membrane becomes increasingly permeable during the later stages of apoptosis, PI can move across the cell membrane and bind to DNA. The stained cells were detected using a flow cytometer (BD Biosciences), and apoptosis was analyzed using ModFit software.

Western blot analysis. Human breast cancer cells were lysed with RIPA lysis buffer (Wolsen, Xi'an, China). Total proteins were measured with the BCA assay. then separated and transferred onto a nitrocellulose membrane (Roche Diagnostics, Basel, Switzerland). A total of $40 \mu \mathrm{g}$ of protein was then isolated by $10 \%$ SDS-PAGE and transferred onto a nitrocellulose membrane (Roche Diagnostics). The membrane was blocked with 5\% non-fat milk in Tris-buffered saline Tween-20 (TBST) for $1 \mathrm{~h}$ at room temperature. The membrane was then incubated with the following primary antibodies: Rabbit polyclonal anti-RASSF9 (PA5-58878, 1:1,000; Invitrogen/Thermo Fisher Scientific, Inc.), rabbit polyclonal anti-p-AKT (sc-135651, 1:1,000), rabbit polyclonal anti-AKT (sc-8312, 1:2,000), mouse monoclonal anti-Cyclin D1 (sc-70899, 1:1,000), mouse monoclonal anti-CDK2 (sc-70829, 1:1,000), rabbit polyclonal anti-p53 (sc-6243, 1:1,000) and mouse monoclonal anti- $\beta$-actin (sc-58673, 1:3,000) (all from Santa Cruz Biotechnology, Inc., Danvers, CA, USA). Next, they were incubated with horseradish peroxidase (HRP)-conjugated secondary antibodies (sc-2005, goat anti-mouse IgG-HRP, 1:5,000; sc-2004, goat anti-rabbit IgG-HRP; Santa Cruz Biotechnology, Inc.) at $37^{\circ} \mathrm{C}$ for $2 \mathrm{~h}$. The membrane was subsequently treated with ECL reagent (Pierce, Rockford, IL, USA) for chemiluminescence detection, and the blots were analyzed using Quantity One imaging software.

Statistical analysis. The data are presented as the means \pm SEM from at least 3 experiments. $\mathrm{P}<0.05$ was considered to indicate a statistically significant difference. Data analysis was conducted using SPSS v.20.0 software (IBM Corporation, Armonk, NY, USA). A Student's t-test was used to analyze the differences between 2 groups. A Chi-square test was employed to analyze the comparisons between miR-1254 expression and certain clinicopathologic characteristics. Correlations between miR-1254 and RASSF9 expression in breast cancer tissues were estimated using Pearson's correlation analysis.

\section{Results}

miR-1254 is frequently upregulated in human breast cancer tissues and cell lines. To examine the function of miR-1254 in breast cancer, we performed RT-qPCR to examine its expression in 79 primary breast carcinoma specimens and 79 adjacent normal breast tissues, and in breast tumor cell lines. The results of RT-qPCR assays revealed that the expression of miR-1254 was markedly increased in $74.7 \%$ (59/79) of the breast cancer tissue samples (a quantitative ratio of $>1.2$ was considered as upregulation) (Fig. 1A and Table I). Further experiments revealed associations between the miR-1254 levels and certain clinicopathological characteristics of the patients with breast cancer. A high miR-1254 expression was associated with tumor size, T stage, TNM stage and estrogen receptor and progesterone receptor positivity (Table I). However, miR-1254 expression was not associated with patient age, lymph node metastasis, tumor grade and human epidermal growth factor receptor 2 positivity. Additionally, miR-1254 expression was significantly upregulated in the breast cancer cell lines (T47D, MCF-7, ZR-75-30 and MDA-MB-231) compared with the HB2 normal breast epithelial cell line (Fig. 1B). These results indicate that miR-1254 may be a useful biomarker for assessing the malignant status of breast cancer.

miR-1254 promotes MCF-7/T47D cell growth and induces G1-S phase transition. The MCF-7/T47D cells were transfected with the miR-1254 precursor expression vector, empty vector, miR-1254 antisense oligonucleotides, or negative control. RT-qPCR was conducted to examine the expression levels of miR-1254 following treatment. Our data indicated that miR-1254 expression was markedly upregulated in the cells transfected with the miR-1254 vector compared with those transfected with the empty vector; however, no significant difference was observed between the anti-miR-1254 and anti-miR-Control groups (Fig. 2A). MTT assays revealed that miR-1254 overexpression facilitated the proliferation of MCF-7/T47D cells at 48 and $72 \mathrm{~h}$ following transfection; however, anti-miR-1254 inhibited MCF-7/T47D cell growth at 48 and $72 \mathrm{~h}$ following transfection (Fig. 2B).

Similar results were observed for cell counting assay. miR-1254 overexpression promoted MCF-7/T47D cell proliferation, while anti-miR-1254 suppressed cell proliferation (Fig. 2C). Since the 
Table I. Association of miR-1254 expression with clinicopathological characteristics of the patients with breast cancer.

\begin{tabular}{|c|c|c|c|c|}
\hline \multirow[b]{2}{*}{ Characteristic } & \multirow[b]{2}{*}{$\begin{array}{l}\text { Number } \\
\text { of cases }\end{array}$} & \multicolumn{2}{|c|}{ miR-1254 expression } & \multirow[b]{2}{*}{ P-value } \\
\hline & & $\begin{array}{l}\text { High } \\
(n=59)\end{array}$ & $\begin{array}{c}\text { Low } \\
(n=20)\end{array}$ & \\
\hline Age & & & & 0.836 \\
\hline$\geq 45$ years & 62 & 46 & 16 & \\
\hline$<45$ years & 17 & 13 & 4 & \\
\hline Tumor size & & & & $0.018^{\mathrm{a}}$ \\
\hline$<20 \mathrm{~mm}$ & 52 & 35 & 17 & \\
\hline$\geq 20 \mathrm{~mm}$ & 27 & 24 & 3 & \\
\hline $\begin{array}{l}\text { Lymph node } \\
\text { metastasis }\end{array}$ & & & & 0.756 \\
\hline Yes & 54 & 40 & 14 & \\
\hline No & 25 & 19 & 6 & \\
\hline Grade & & & & 0.907 \\
\hline 1 & 23 & 18 & 5 & \\
\hline 2 & 41 & 30 & 11 & \\
\hline 3 & 15 & 11 & 4 & \\
\hline T stage & & & & $0.008^{\mathrm{a}}$ \\
\hline $\mathrm{T} 1$ & 22 & 12 & 10 & \\
\hline $\mathrm{T} 2$ & 41 & 33 & 8 & \\
\hline $\mathrm{T} 3$ & 7 & 6 & 1 & \\
\hline $\mathrm{T} 4$ & 9 & 8 & 1 & \\
\hline TNM stage & & & & $0.026^{\mathrm{a}}$ \\
\hline I & 24 & 16 & 8 & \\
\hline II & 33 & 24 & 9 & \\
\hline III & 12 & 10 & 2 & \\
\hline IV & 10 & 9 & 1 & \\
\hline ER & & & & $0.003^{\mathrm{a}}$ \\
\hline Positive & 51 & 45 & 6 & \\
\hline Negative & 28 & 14 & 14 & \\
\hline PR & & & & $0.031^{\mathrm{a}}$ \\
\hline Positive & 46 & 38 & & \\
\hline Negative & 33 & 21 & 12 & \\
\hline HER2 & & & & $0.452^{\mathrm{a}}$ \\
\hline Positive & 30 & 23 & 7 & \\
\hline Negative & 49 & 36 & 13 & \\
\hline
\end{tabular}

${ }^{\mathrm{a}} \mathrm{P}<0.05$. ER, estrogen receptor; $\mathrm{PR}$, progesterone receptor; HER2, human epidermal growth factor receptor 2.

cell cycle involves the regulation of proliferation, we analyzed it following transfection. We found that miR-1254 overexpression decreased the G1/G0 phase population and increased the $S$ phase population in the MCF-7/T47D cells; however, the inhibition of miR-1254 led to a marked accumulation of cells in the G1/G0 phase and a reduction of the $\mathrm{S}$ phase cell population (Fig. 2D). Apoptosis analysis revealed that the proportion of cells undergoing early and late stage apoptosis decreased markedly in the miR-1254 overexpression group, while it increased significantly in the
Table II. Association of RASSF9 mRNA expression with the clinicopathological characteristics of patients with breast cancer.

\begin{tabular}{|c|c|c|c|c|}
\hline \multirow[b]{2}{*}{ Characteristics } & \multirow[b]{2}{*}{$\begin{array}{l}\text { Number } \\
\text { of cases }\end{array}$} & \multicolumn{2}{|c|}{$\begin{array}{c}\text { RASSF9 mRNA } \\
\text { expression }\end{array}$} & \multirow[b]{2}{*}{ P-value } \\
\hline & & $\begin{array}{l}\text { High } \\
(n=16)\end{array}$ & $\begin{array}{c}\text { Low } \\
(n=63)\end{array}$ & \\
\hline Age & & & & 0.803 \\
\hline$\geq 45$ years & 62 & 12 & 50 & \\
\hline$<45$ years & 17 & 4 & 13 & \\
\hline Tumor size & & & & $0.009^{\mathrm{a}}$ \\
\hline$<20 \mathrm{~mm}$ & 52 & 14 & 38 & \\
\hline$\geq 20 \mathrm{~mm}$ & 27 & 2 & 25 & \\
\hline $\begin{array}{l}\text { Lymph node } \\
\text { metastasis }\end{array}$ & & & $0.015^{\mathrm{a}}$ & \\
\hline Yes & 54 & 5 & 49 & \\
\hline No & 25 & 11 & 14 & \\
\hline Grade & & & & 0.637 \\
\hline 1 & 23 & 5 & 18 & \\
\hline 2 & 41 & 8 & 33 & \\
\hline 3 & 15 & 3 & 12 & \\
\hline T stage & & & & $0.023^{\mathrm{a}}$ \\
\hline $\mathrm{T} 1$ & 22 & 7 & 15 & \\
\hline $\mathrm{T} 2$ & 41 & 8 & 33 & \\
\hline T3 & 7 & 1 & 6 & \\
\hline $\mathrm{T} 4$ & 9 & 0 & 9 & \\
\hline TNM stage & & & & $0.013^{\mathrm{a}}$ \\
\hline I & 24 & 5 & 19 & \\
\hline II & 33 & 8 & 25 & \\
\hline III & 12 & 2 & 10 & \\
\hline IV & 10 & 1 & 9 & \\
\hline ER & & & & $0.001^{\mathrm{a}}$ \\
\hline Positive & 51 & 3 & 48 & \\
\hline Negative & 28 & 13 & 15 & \\
\hline PR & & & & $0.045^{\mathrm{a}}$ \\
\hline Positive & 46 & 6 & 40 & \\
\hline Negative & 33 & 10 & 23 & \\
\hline HER2 & & & & 0.118 \\
\hline Positive & 30 & 4 & 26 & \\
\hline Negative & 49 & 12 & 37 & \\
\hline
\end{tabular}

${ }^{\mathrm{a}} \mathrm{P}<0.05$. ER, estrogen receptor; $\mathrm{PR}$, progesterone receptor; HER2, human epidermal growth factor receptor 2 .

anti-miR-1254 group (Fig. 2E). These data suggest that miR-1254 promotes breast cancer cell proliferation and G1-S transition, and inhibits cell apoptosis.

RASSF9 is a direct target of $m i R-1254$. miRanda and RegRNA were used to predict many possible target genes of miR-1254. Among the candidates, RASSF9 was selected for further 

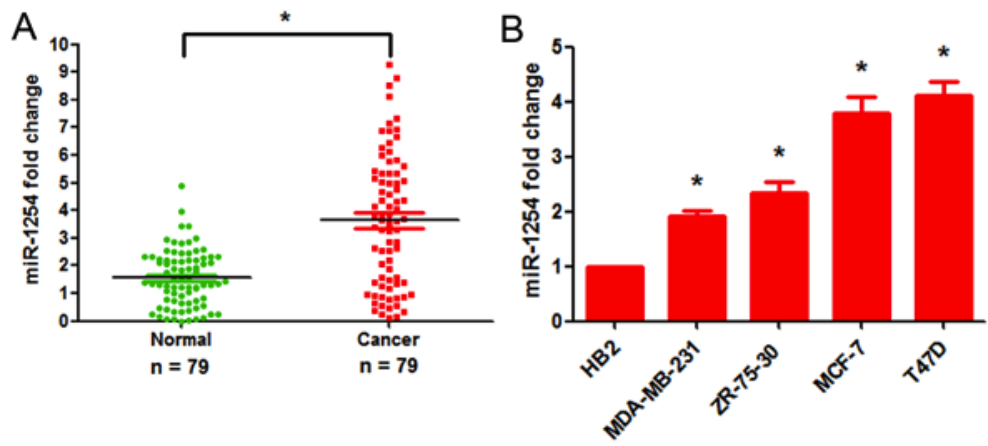

Figure 1. miR-1254 is upregulated in breast cancer tissues and cell lines. (A) RT-qPCR assays revealed that miR-1254 expression was significantly increased in breast cancer tissues, as compared with in normal breast tissues ( $\left.\mathrm{n}=79,{ }^{*} \mathrm{P}<0.001\right)$. (B) RT-qPCR was performed to analyze miR-1254 expression in the breast cancer cell lines, T47D, MCF-7, ZR-75-30, MDA-MB-231, and in a normal breast epithelial cell line (HB2) ( $\mathrm{P}<0.01)$.
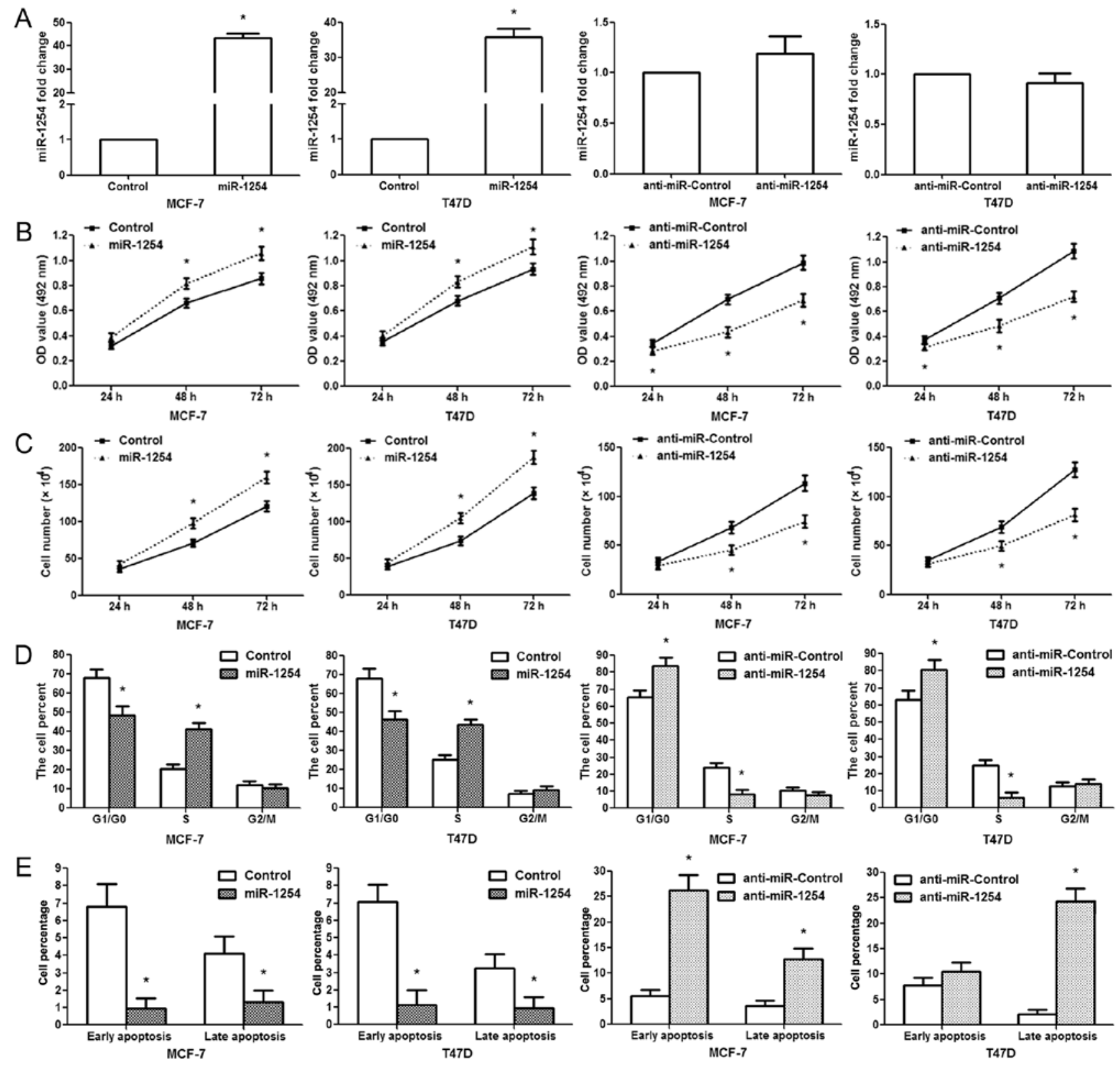

Figure 2. miR-1254 increases MCF-7/T47D cell proliferation and induces G1-S phase transition. (A) miR-1254 expression was assessed in MCF-7/T47D cells following miR-1254 overexpression or anti-miR-1254 treatment. (B) MTT assays revealed that miR-1254 overexpression accelerated cell activity, while anti-miR-1254 reduced cell activity at 48 and $72 \mathrm{~h}$ following transfection. (C) Cell counting assays revealed that miR-1254 overexpression promoted MCF-7/T47D cell proliferation and that anti-miR-1254 inhibited cell growth. (D) Flow cytometric analysis of the cell cycle. Histograms represent the proportions of G1/G0, S and G2/M phase cells following transfection. (E) Flow cytometric analysis of cell apoptosis. Histograms represent the proportions cells in early and late apoptosis. $\mathrm{n}=3$ experiments, ${ }^{*} \mathrm{P}<0.01$. 
A
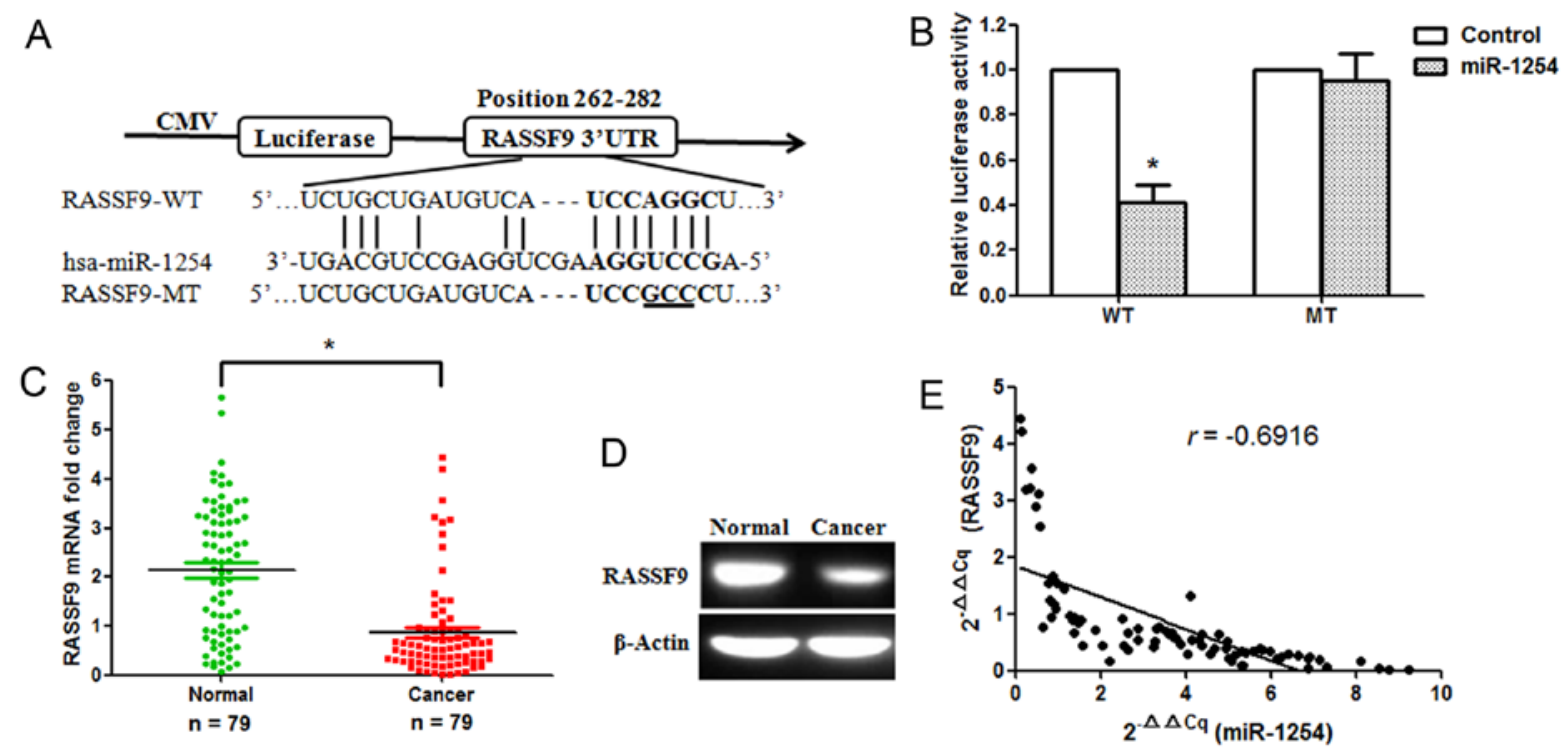

$\mathrm{F}$
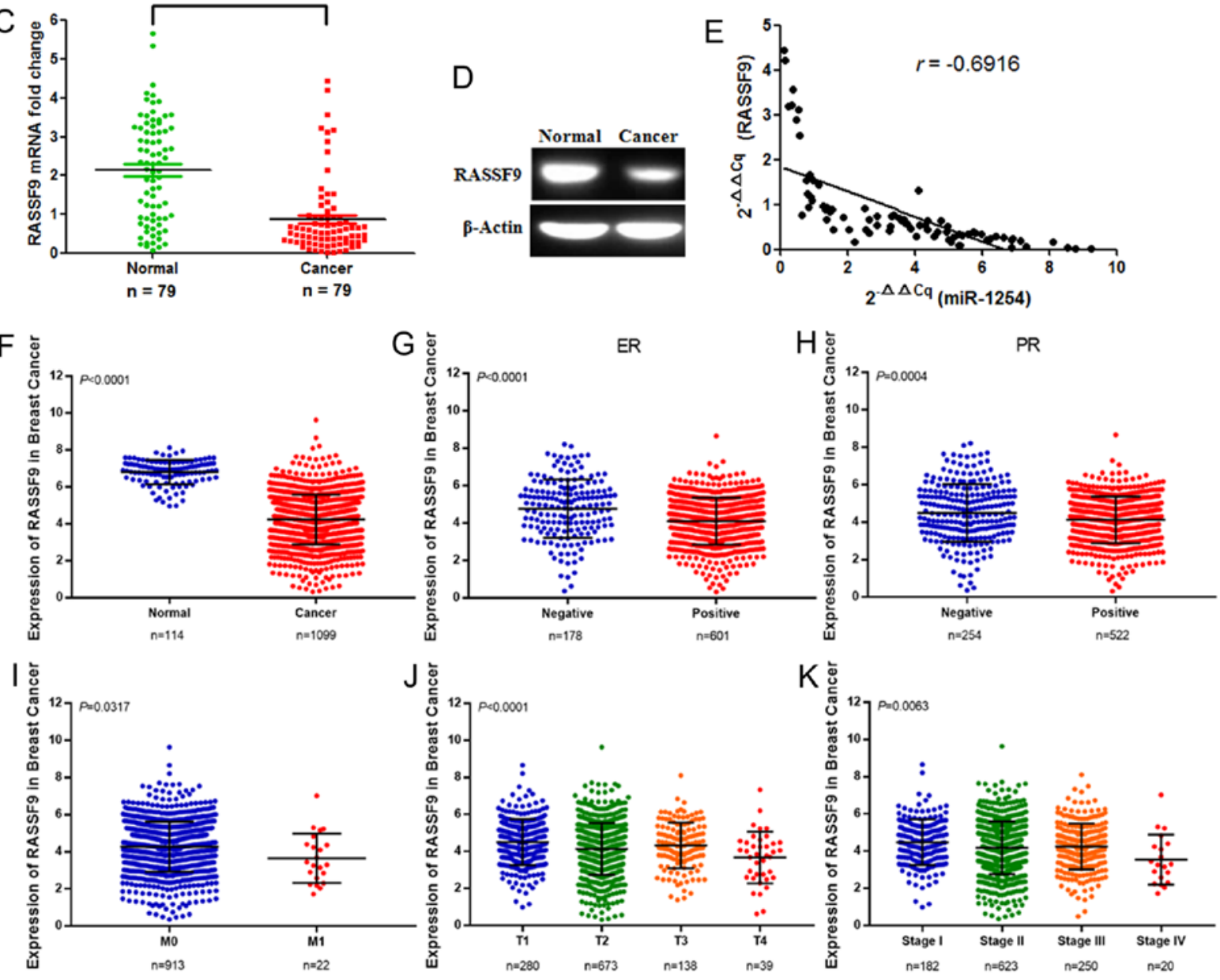

Figure 3. miR-1254 directly targets the RASSF9 gene. (A) The binding site of miR-1254 at the 3'-UTR of RASSF9 was predicted by bioinformatics (B) Luciferase reporter vectors, including wild- or mutant-type RASSF9-3'-UTR, were co-transfected into 293T cells in combination with miR-1254 or Control. Luciferase activity was examined with a dual luciferase assay; ${ }^{*} \mathrm{P}<0.01$. (C) RASSF9 mRNA expression in breast cancer tissues; ${ }^{*} \mathrm{P}<0.001$. (D) RASSF9 protein expression was detected by western blot analysis. (E) miR-1254 and RASSF9 expression levels exhibited a negative correlation. miR-1254 and RASSF9 expression levels were analyzed by Pearson's correlation analysis ( $n=79, r=-0.6916, P<0.001$, Pearson's correlation). (F) RASSF9 expression in patients with breast cancer using data from TCGA. (G) RASSF9 expression and estrogen receptor positivity in breast cancer tissues using data from TCGA. (H) RASSF9 expression and progesterone receptor positivity in breast cancer tissues using data from TCGA. (I) RASSF9 expression and M stage in breast cancer tissues using data from TCGA. (J) RASSF9 expression and T stage in breast cancer tissues using data from TCGA. (K) RASSF9 expression and TNM stage in breast cancer tissues using data from TCGA.

research. We discovered a binding site for miR-1254 in the 3'-UTR of RASSF9 mRNA ranging from 262-282 bp (Fig. 3A). To confirm whether miR-1254 directly targets RASSF9, a dual-luciferase reporter assay, including the WT and MT 3'-UTRs of RASSF9, was performed. Reporter plasmids and pre-miR-1254 or pmirGLO control vector (Control) were co-transfected into $293 \mathrm{~T}$ cells. The luciferase activity of the pre-miR-1254/WT-RASSF9-UTR-transfected cells decreased significantly, but that of the pre-miR-1254/MT-RASSF9-UTRtransfected cells failed to suppress the relative luciferase activity (Fig. 3B), suggesting that miR-1254 can bind to the 3'-UTR of RASSF9. Subsequently, the RASSF9 mRNA and protein expression levels were measured. The results revealed that RASSF9 expression was significantly downregulated in the breast cancer tissues (Fig. 3C and D). The effect of miR-1254 on RASSF9 expression was assessed based on the results of RT-qPCR. A significantly negative correlation was identified between RASSF9 and miR-1254 (Fig. 3E; n=79, r=-0.6916, $\mathrm{P}<0.001$, Pearson's correlation analysis). The TCGA data revealed that RASSF9 expression was markedly downregulated in breast cancer tissues. A low RASSF9 expression was associated with estrogen receptor positivity, progesterone receptor positivity, $M$ stage, $T$ stage and TNM stage (Fig. 3F-K). In addition, to verify the changes in RASSF9 expression in breast 

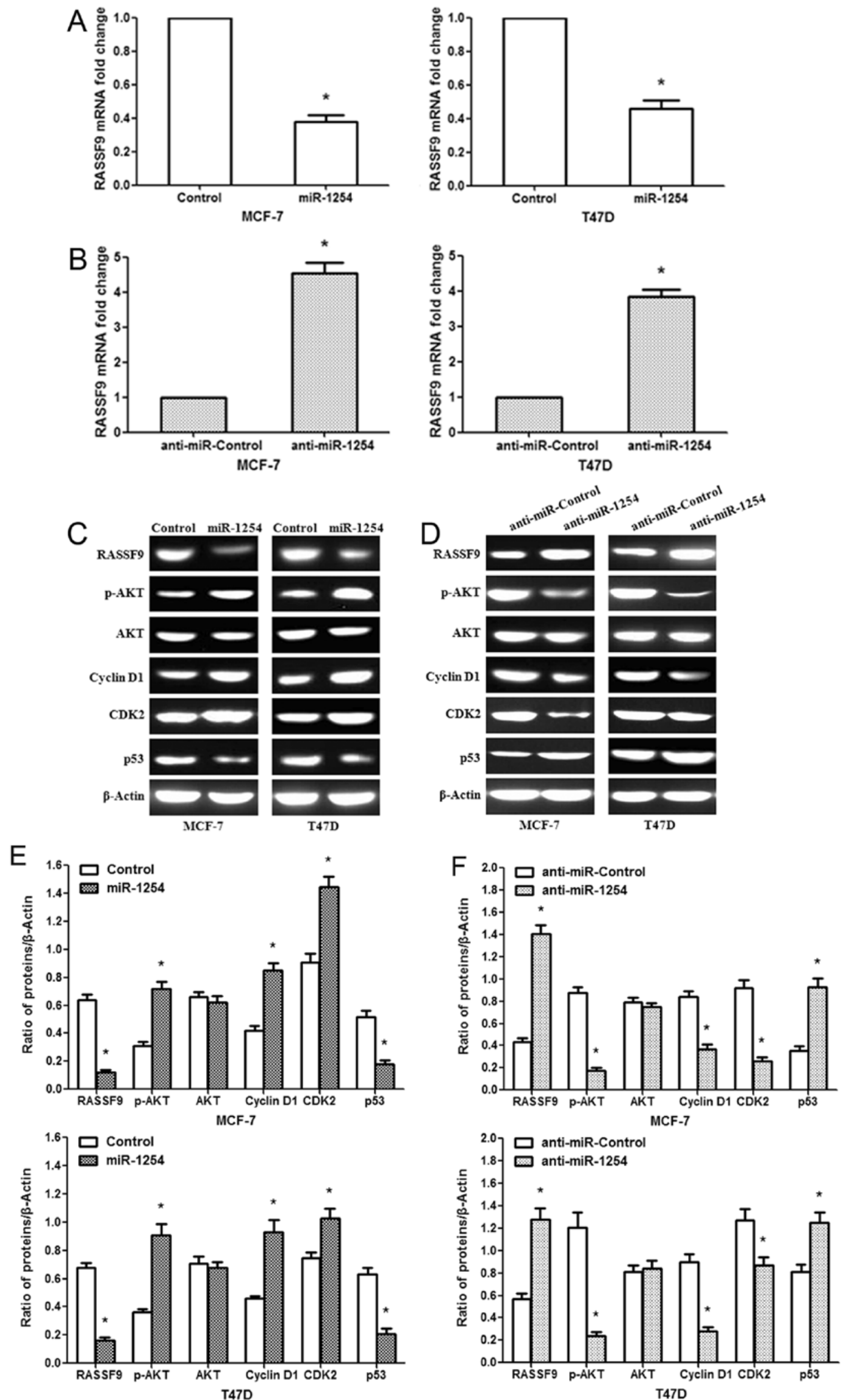

Figure 4. miR-1254 regulates the AKT and p53 signaling pathways in breast cancer by targeting RASSF9. (A) RASSF9 mRNA was determined in MCF-7/T47D cells following miR-1254 overexpression. ${ }^{*} \mathrm{P}<0.01$. (B) RASSF9 mRNA was measured in MCF-7/T47D cells following anti-miR-1254 treatment; ${ }^{*} \mathrm{P}<0.001$. (C) miR-1254 overexpression promoted the expression of p-AKT, Cyclin D1 and CDK2, and inhibited RASSF9 and p53 expression in MCF-7/T47D cells. (D) Anti-miR-1254 suppressed p-AKT, Cyclin D1 and CDK2 expression, and increased RASSF9 and p53 expression. (E) Densitometric analysis of changes in protein expression following miR-1254 treatment; ${ }^{*} \mathrm{P}<0.01$. (F) Densitometric analysis of changes in protein expression following anti-miR-1254 treatment; ${ }^{*} \mathrm{P}<0.01$. 

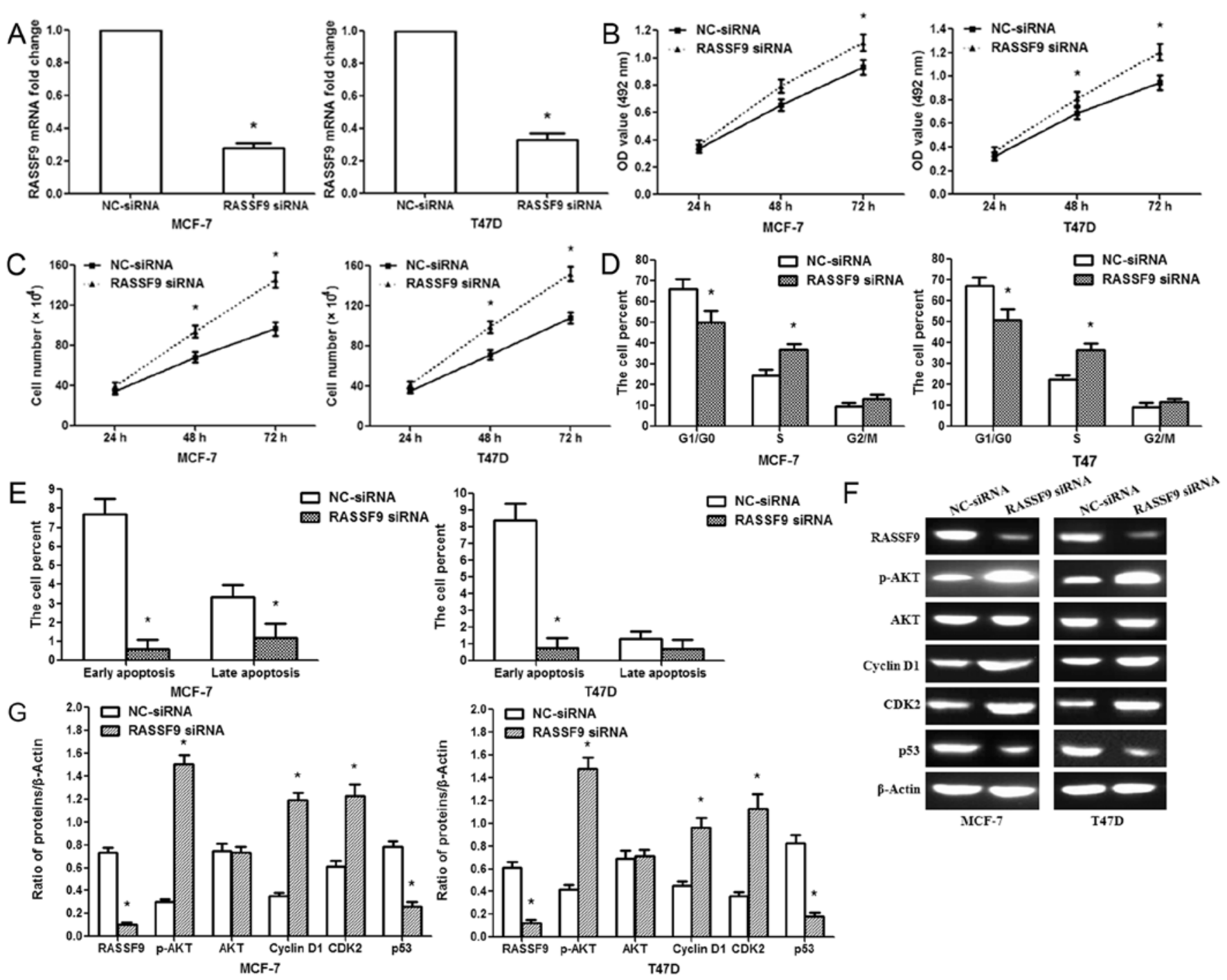

Figure 5. RASSF9 siRNA promotes the growth of breast cancer MCF-7/T47D cells. (A) RT-qPCR revealed the knockdown efficiency of RASSF9 siRNA in MCF-7/T47D cells. (B) MTT assays revealed that RASSF9 siRNA promoted the activity of MCF-7/T47D cells at 48 and $72 \mathrm{~h}$. (C) Cell counting assays revealed that RASSF9 siRNA promoted MCF-7/T47D cell proliferation. (D) Flow cytometric analysis revealed the percentage of MCF-7/T47D cells in each cell cycle phase. G1/G0 phase cells decreased after RASSF9 siRNA treatment, and S phase cells increased. (E) Percentage of cells in early and late apoptosis. (F) RASSF9, p-AKT, AKT, Cyclin D1, CDK2 and p53 protein expression levels were examined following transfection with RASSF9 siRNA. (G) Densitometric analysis of changes in protein expression following RASSF9 siRNA treatment. " $\mathrm{P}<0.01$, compared with the NC-siRNA group, $\mathrm{n}=3$.

cancer tissues, its mRNA levels were detected in the 79 breast cancer tissues by RT-qPCR. A low RASSF9 expression was found to be associated with tumor size, lymph node metastasis, $\mathrm{T}$ stage, TNM stage, estrogen receptor positivity and progesterone receptor positivity (Table II).

In addition, we found that miR-1254 overexpression markedly downregulated the mRNA expression of RASSF9 in MCF-7/T47D cells, while anti-miR-1254 increased RASSF9 mRNA expression (Fig. 4A and B). Similar results were observed for the protein levels (Fig. 4C and D). To further explore the potential mechanisms of the miR-1254-regulated cell proliferation, cell cycle transition and apoptosis, we measured protein expression in the AKT signaling pathway, and that of $\mathrm{G} 1$ phase regulators and $\mathrm{p} 53$. The data revealed that miR-1254 overexpression increased p-AKT, Cyclin D1 and CDK2 protein expression, and decreased the p53 protein levels in the MCF-7/T47D cells (Fig. 4C). By contrast, anti-miR-1254 inhibited p-AKT, Cyclin D1 and CDK2 protein expression, and promoted p53 expression (Fig. 4D). These results indicate that miR-1254 may modulate breast cancer cell proliferation and apoptosis through the regulation of the AKT and p53 signaling pathways by targeting RASSF9.

Knockdown of RASSF9 facilitates MCF-7/T47D cell proliferation. We knocked down RASSF9 expression in the MCF-7/T47D cells via RNA interference to affirm its involvement in the pro-tumor functions of miR-1254. RASSF9 mRNA expression was specifically knocked down in the MCF-7/T47D cells using siRNA (Fig. 5A). The silencing of RASSF9 significantly increased cell activity at 48 and $72 \mathrm{~h}$ following treatment (Fig. 5B). A cell counting assay revealed that the silencing of RASSF9 promoted MCF-7/T47D cell proliferation (Fig. 5C). The silencing of RASSF9 decreased the G1/G0 phase population and increased the $S$ phase population of the MCF-7/T47D cells (Fig. 5D). Furthermore, the silencing of RASSF9 inhibited the apoptosis of MCF-7/T47D 

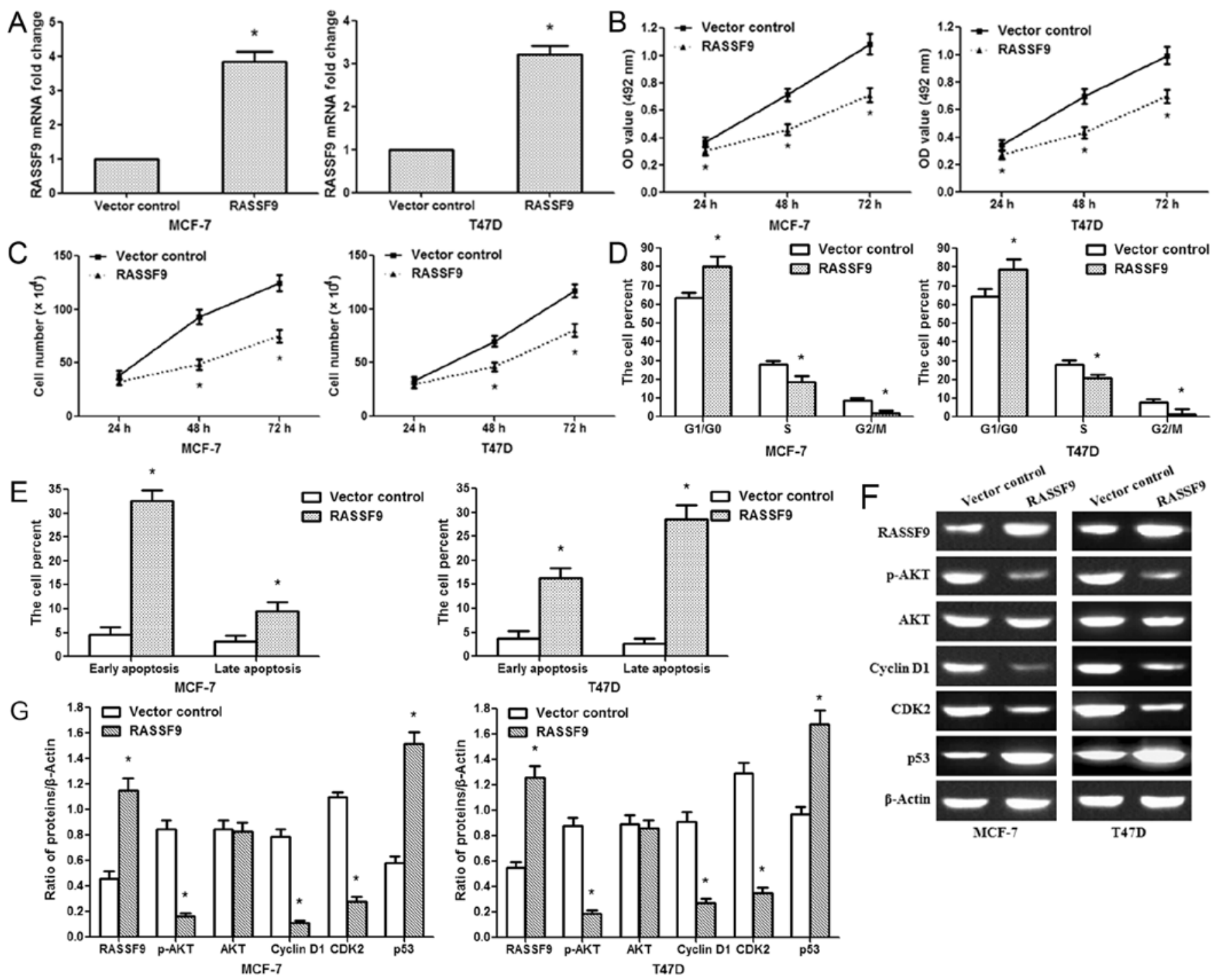

Figure 6. RASSF9 overexpression inhibits the proliferation of breast cancer MCF-7/T47D cells. (A) RASSF9 overexpression increased RASSF9 mRNA levels in MCF-7/T47D cells. (B) MTT revealed that RASSF9 overexpression reduced MCF-7/T47D cell activity at 48 and $72 \mathrm{~h}$. (C) Cell counting assay showed that RASSF9 overexpression suppressed MCF-7/T47D cell proliferation. (D) Flow cytometric analysis revealed the percentage of MCF-7/T47D cells in each cell cycle phase. (E) Proportion of cells in early and late apoptosis following transfection with RASSF9 overexpression vector. (F) RASSF9, p-AKT, AKT, Cyclin D1, CDK2 and p53 protein expression levels were examined following transfection with RASSF9 overexpression vector. (G) Densitometric analysis of changes in protein expression following RASSF9 overexpression vector treatment. ${ }^{*} \mathrm{P}<0.01$, compared with the Vector control group, $\mathrm{n}=3$.

cells (Fig. 5E). These effects were similar to those caused by miR-1254 overexpression, suggesting a similar effect caused by both RASSF9 knockdown and miR-1254 overexpression. In addition, we examined the knockdown efficiency of RASSF9 siRNA. RASSF9 protein expression decreased in the siRNA group; p53 protein expression was also decreased. However, p-AKT, Cyclin D1 and CDK2 protein expression increased in the siRNA group (Fig. 5F).

Overexpression of RASSF9 inhibits MCF-7/T47D cell proliferation. We then constructed a RASSF9 overexpression plasmid. In the MCF-7/T47D cells, the plasmid efficiently upregulated the RASSF9 mRNA levels (Fig. 6A). Based on the results of cell viability and cell counting assays, the upregulation of RASSF9 inhibited MCF-7/T47D cell growth (Fig. 6B and C). The impact of RASSF9 expression on the cell cycle was measured by flow cytometry, and it was found that RASSF9 overexpression increased the G1 phase cell proportion and decreased the $\mathrm{S}$ and G2/M phase cell proportion (Fig. 6D). As for the effect of RASSF9 expression on cell apoptosis, RASSF9 overexpression significantly induced early and late apoptosis (Fig. 6E). Further analysis revealed that RASSF9 overexpression inhibited G1-S phase transition by suppressing p-AKT, Cyclin D1 and CDK2 expression. Additionally, it promoted the expression of key apoptosis regulators, such as p53 (Fig. 6F). Therefore, these data indicate that miR-1254 regulates breast cancer cell progression by targeting RASSF9 through the AKT and p53 signaling pathways.

\section{Discussion}

Previous studies have demonstrated that miRNAs play crucial roles in regulating tumor cell survival, proliferation, apoptosis, differentiation, migration and invasion (9,34). It has been found that abnormally expressed miRNAs are associated with breast carcinogenesis and progression $(35,36)$. Although miRNA 
profiles have been well-characterized in breast cancer, the roles and mechanisms of action of dysregulated miRNAs remain largely unknown. Authenticating miRNAs and elucidating their biological effects in breast cancer is crucial for identifying novel targets for diagnosis and treatment. It has been reported that miR-1254 expression is increased in lung cancer and promotes lung cancer cell proliferation by targeting SFRP1 (18). However, miR-1254 has been shown to inhibit oral squamous cell carcinoma metastasis (20). Nevertheless, the clinical implication and function of miR-1254 in breast cancer remains unclear. In the present study, we found that miR-1254 expression was markedly upregulated in breast cancer tissues and cell lines. The results revealed that a high miR-1254 expression was associated with tumor size, T stage, TNM stage, estrogen receptor positivity and progesterone receptor positivity in breast cancer. The results demonstrated that miR-1254 overexpression promoted breast cancer cell growth by inducing G1-S phase transition and inhibiting cell apoptosis in vitro. Anti-miR-1254 inhibited breast cancer cell growth and induced cell apoptosis, whereas there was no significant difference between the antimiR-1254 and anti-miR-Control groups. This is due to the fact that the inhibitor may suppress the function of miR-1254 by binding to it, but does not affect the expression of miR-1254. These findings suggest that miR-1254 plays a crucial role in breast cancer development and progression.

RASSF9-null mice exhibit a marked variety in epithelial organization, including cell proliferation and differentiation (37). In this study, to the best of our knowledge, we present the first evidence that miR-1254 promotes breast cancer cell proliferation through the miR-1254 target, RASSF9. RASSF9 was predicted to be a target of miR-1254 by two databases. The results of reporter assays revealed that miR-1254 overexpression inhibited RASSF9 3'-UTR luciferase reporter activity, which was abrogated by the mutation of the miR-1254 binding site; we also found a negative correlation between miR-1254 and RASSF9 expression in breast carcinoma tissues; miR-1254 overexpression inhibited RASSF9 mRNA and protein expression in breast cancer cells. Our findings also demonstrated that the knockdown of RASSF9 promoted breast cancer cell proliferation and suppressed apoptosis, and that the overexpression of RASSF9 inhibited cell proliferation and induced apoptosis. These results suggest that miR-1254 acts as a positive regulator or oncogene for cell proliferation, partially mediated by inhibiting RASSF9 expression.

$\mathrm{PI} 3 \mathrm{~K} / \mathrm{AKT}$ is one of the most effective growth signaling pathways in cancer (38). Aberrations in the AKT signaling pathway are involved in tumorigenesis and progression, particularly in breast, liver, lung, prostate, colorectal and renal cancer (39). AKT activity is reportedly associated with various clinicopathological characteristics of cancer (40). AKT regulates the roles of certain substrates related to the cell cycle by directly phosphorylating target proteins or indirectly controlling protein expression (39). The downstream regulated genes of AKT, Cyclin D1 and CDK2, are crucial transcriptional factors in the G0/G1 phase (41). Cyclin A-CDK2 and Cyclin D-CDK4/6 protein kinase complexes, important cell cycle regulators, regulate progression from the G1/G0 to the $S$ phase (42). D-Cyclins drive entry into the $S$ phase by releasing E2F transcription factors following extracellular mitogenic stimulation. Cyclin A-CDK2 protein kinase complexes regulate proliferation and the cell cycle in lung and renal cancer $(43,44)$. The findings of the present study demonstrate that miR-1254 overexpression and RASSF9 siRNA can promote Cyclin D1 expression and drive more cells into the $\mathrm{S}$ phase by activating the AKT signaling pathway, while antimiR-1254 and RASSF9 overexpression may inhibit Cyclin D1 expression and induce G1-phase arrest by suppressing the AKT signaling pathway. These results suggest that miR-1254 may promote the expression of Cyclin D1 and induce G1-S phase transition by activating the AKT signaling pathway via targeting RASSF9.

p53 is a transcription factor able to regulate the expression of numerous downstream target genes responsible for directly or indirectly controlling senescence, apoptosis, DNA repair and genetic stability in response to various cellular stresses (45). The results of this study indicated that miR-1254 overexpression or RASSF9 siRNA inhibited breast cancer cell apoptosis by suppressing p53 expression; anti-miR-1254 and RASSF9 overexpression induced apoptosis by promoting p53 expression. These results indicate that miR-1254 may suppress breast cancer cell apoptosis by regulating p53 expression via RASSF9.

In conclusion, this study demonstrated that miR-1254 functions as an oncogene in breast cancer. We found that miR-1254 was both upregulated and associated with certain clinicopathological characteristics of patients with breast cancer. miR-1254 promoted breast cancer cell proliferation by activating the AKT signaling pathway and suppressed apoptosis through the regulation of p53 expression via RASSF9. These findings indicate that miR-1254 plays a crucial role in breast cancer progression and may thus represent a latent novel target for breast cancer therapy.

\section{Acknowledgements}

Not applicable.

\section{Funding}

This study was supported by the Shaanxi Province Natural Science Foundation (grant no. 2016JM4263) and the China Postdoctoral Science Foundation (grant no. 2016M546537).

\section{Availability of data and materials}

All data generated or analyzed during this study are included in this published article.

\section{Authors' contributions}

BL and $\mathrm{JH}$ designed the experiments. PC and JW collected the clinical data and samples. BL, PC, JW, LW, MR and RZ performed the experiments. BL and JW performed the statistical analysis. BL, LW and $\mathrm{JH}$ wrote and edited the manuscript. JH supervised the study. All authors have read and approved the final manuscript.

\section{Ethics approval and consent to participate}

For the use of patient samples, informed consent was obtained from each patient prior to specimen collection. The study 
protocol was approved by the Ethics Committee of $\mathrm{Xi}^{\prime}$ an Jiaotong University (Xi'an, China).

\section{Patient consent for publication}

Not applicable.

\section{Competing interests}

The authors declare that they have no competing interests.

\section{References}

1. Obayashi S, Horiguchi J, Higuchi T, Katayama A, Handa T, Altan B, Bai T, Bao P, Bao H, Yokobori T, et al: Stathmin1 expression is associated with aggressive phenotypes and cancer stem cell marker expression in breast cancer patients. Int J Oncol 51: 781-790, 2017.

2. Siegel RL, Miller KD and Jemal A: Cancer statistics, 2016. CA Cancer J Clin 66: 7-30, 2016.

3. Fan L, Strasser-Weippl K, Li JJ, St Louis J, Finkelstein DM, Yu KD, Chen WQ, Shao ZM and Goss PE: Breast cancer in China. Lancet Oncol 15: e279-e289, 2014.

4. Espinoza-Sánchez NA, Vadillo E, Balandrán JC, MonroyGarcía A, Pelayo R and Fuentes-Pananá EM: Evidence of lateral transmission of aggressive features between different types of breast cancer cells. Int J Oncol 51: 1482-1496, 2017.

5. Zhao L, Liu Y, Tong D, Qin Y, Yang J, Xue M, Du N, Liu L, Guo B, Hou N, et al: MeCP2 promotes gastric cancer progression through regulating FOXF1/Wnt5a/ $\beta$-Catenin and MYOD1/ Caspase-3 signaling pathways. EBioMedicine 16: 87-100, 2017.

6. Raman V, Fuentes Lorenzo JL, Stashenko EE, Levy M, Levy MM and Camarillo IG: Lippia origanoides extract induces cell cycle arrest and apoptosis and suppresses $\mathrm{NF}-\kappa \mathrm{B}$ signaling in triplenegative breast cancer cells. Int J Oncol 51: 1801-1808, 2017.

7. Bhardwaj A, Rosen D, Liu M, Liu Y, Hao Q, Ganesan N, Etzel CJ, Gullett A, Albarracin CT and Bedrosian I: Suppression of Akt-mTOR pathway-a novel component of oncogene induced DNA damage response barrier in breast tumorigenesis. PLoS One 9: e97076, 2014.

8. Byler S, Goldgar S, Heerboth S, Leary M, Housman G, Moulton K and Sarkar S: Genetic and epigenetic aspects of breast cancer progression and therapy. Anticancer Res 34: 1071-1077, 2014.

9. Zhao LY, Tong DD, Xue M, Ma HL, Liu SY, Yang J, Liu YX, Guo B, Ni L, Liu LY, et al: MeCP2, a target of miR-638, facilitates gastric cancer cell proliferation through activation of the MEK1/2-ERK1/2 signaling pathway by upregulating GIT1 Oncogenesis 6: e368, 2017.

10. Hyrina A, Olmstead AD, Steven P, Krajden M, Tam E and Jean F: Treatment-induced viral cure of hepatitis c virus-infected patients involves a dynamic interplay among three important molecular players in lipid homeostasis: Circulating microRNA (miR)-24, miR-223, and proprotein convertase subtilisin/kexin type 9. EBioMedicine 23: 68-78, 2017.

11. Zhao LY, Yao Y, Han J, Yang J, Wang XF, Tong DD, Song TS, Huang $\mathrm{C}$ and Shao Y: miR-638 suppresses cell proliferation in gastric cancer by targeting Sp2. Dig Dis Sci 59: 1743-1753, 2014

12. Xu J, Lin H, Li G, Sun Y, Chen J, Shi L, Cai X and Chang C The miR-367-3p increases sorafenib chemotherapy efficacy to suppress hepatocellular carcinoma metastasis through altering the androgen receptor signals. EBioMedicine 12: 55-67, 2016.

13. Selbach M, Schwanhäusser B, Thierfelder N, Fang Z, Khanin R and Rajewsky N: Widespread changes in protein synthesis induced by microRNAs. Nature 455: 58-63, 2008.

14. Shi M, Liu D, Shen B and Guo N: Helpers of the cellular gatekeeper-miRNAs dance in P53 network. Biochim Biophys Acta 1805: 218-225, 2010.

15. Sahu A, Jha PK, Prabhakar A, Singh HD, Gupta N, Chatterjee T, Tyagi T, Sharma S, Kumari B, Singh S, et al: MicroRNA-145 impedes thrombus formation via targeting tissue factor in Venous thrombosis. EBioMedicine 26: 175-186, 2017.

16. Zheng Y, Lv X, Wang X, Wang B, Shao X, Huang Y, Shi L, Chen Z, Huang J and Huang P: MiR-181b promotes chemoresistance in breast cancer by regulating Bim expression. Oncol Rep 35: 683-690, 2016.
17. Ji Y, Han Z, Shao L and Zhao Y: Evaluation of in vivo antitumor effects of low-frequency ultrasound-mediated miRNA-133a microbubble delivery in breast cancer. Cancer Med 5: 2534-2543, 2016.

18. Li H, Yang T, Shang D and Sun Z: miR-1254 promotes lung cancer cell proliferation by targeting SFRP1. Biomed Pharmacother 92: 913-918, 2017.

19. Li Q, Shen W, Li X, Zhang L and Jin X: The lncRNA n340790 accelerates carcinogenesis of thyroid cancer by regulating miR-1254. Am J Transl Res 9: 2181-2194, 2017.

20. Lu M, Chen WH, Wang CY, Mao CQ and Wang J: Reciprocal regulation of miR-1254 and c-Myc in oral squamous cell carcinoma suppresses EMT-mediated metastasis and tumorinitiating properties through MAPK signaling. Biochem Biophys Res Commun 484: 801-807, 2017.

21. Sherwood V, Recino A, Jeffries A, Ward A and Chalmers AD: The N-terminal RASSF family: A new group of Ras-associationdomain-containing proteins, with emerging links to cancer formation. Biochem J 425: 303-311, 2010.

22. Zheng X, Dong Q, Zhang X, Han Q, Han X, Han Y, Wu J, Rong X and Wang E: The coiled-coil domain of oncogene RASSF 7 inhibits hippo signaling and promotes non-small cell lung cancer. Oncotarget 8: 78734-78748, 2017.

23. Lock FE, Underhill-Day N, Dunwell T, Matallanas D, Cooper W, Hesson L, Recino A, Ward A, Pavlova T, Zabarovsky E, et al: The RASSF8 candidate tumor suppressor inhibits cell growth and regulates the Wnt and NF- $\mathrm{BB}$ signaling pathways. Oncogene 29: 4307-4316, 2010

24. Wang L, Liu W, Zhang YP and Huang XR: The miR-224 promotes non-small cell lung cancer cell proliferation by directly targeting RASSF8. Eur Rev Med Pharmacol Sci 21: 3223-3231, 2017.

25. He C, Wang L, Zhang J and $\mathrm{Xu} \mathrm{H}$ : Hypoxia-inducible microRNA-224 promotes the cell growth, migration and invasion by directly targeting RASSF8 in gastric cancer. Mol Cancer 16: $35,2017$.

26. Huang Y, Li Y, Wang FF, Lv W, Xie X and Cheng X: Overexpressed miR-224 promotes the progression of cervical cancer via targeting RASSF8. PLoS One 11: e0162378, 2016.

27. Hesson LB, Dunwell TL, Cooper WN, Catchpoole D, Brini AT, Chiaramonte R, Griffiths M, Chalmers AD, Maher ER and Latif F: The novel RASSF6 and RASSF10 candidate tumour suppressor genes are frequently epigenetically inactivated in childhood leukaemias. Mol Cancer 8: 42, 2009.

28. Schagdarsurengin U, Richter AM, Wohler C and Dammann RH: Frequent epigenetic inactivation of RASSF10 in thyroid cancer. Epigenetics 4: 571-576, 2009.

29. Liu W, Wang J, Wang L, Qian C, Qian Y, Xuan H, Zhuo W, Li X, Yu J and Si J: Ras-association domain family 10 acts as a novel tumor suppressor through modulating MMP2 in hepatocarcinoma. Oncogenesis 5: e237, 2016.

30. Richter AM, Walesch SK and Dammann RH: aberrant promoter methylation of the tumour suppressor RASSF10 and its growth inhibitory function in breast cancer. Cancers (Basel) 8: 26, 2016.

31. Li X, Liang Q, Liu W, Zhang N, Xu L, Zhang X, Zhang J, Sung JJ and $\mathrm{Yu}$ J: Ras association domain family member 10 suppresses gastric cancer growth by cooperating with GSTP1 to regulate JNK/c-Jun/AP-1 pathway. Oncogene 35: 2453-2464, 2016.

32. Lu D, Ma J, Zhan Q, Li Y, Qin J and Guo M: Epigenetic silencing of RASSF10 promotes tumor growth in esophageal squamous cell carcinoma. Discov Med 17: 169-178, 2014.

33. Livak KJ and Schmittgen TD: Analysis of relative gene expression data using real-time quantitative PCR and the 2(-Delta Delta C(T)) methods. Methods 25: 402-408, 2001.

34. Padi SK, Zhang Q, Rustum YM, Morrison C and Guo B: MicroRNA-627 mediates the epigenetic mechanisms of vitamin D to suppress proliferation of human colorectal cancer cells and growth of xenograft tumors in mice. Gastroenterology 145: 437-446, 2013

35. Gong C, Tan W, Chen K, You N, Zhu S, Liang G, Xie X, Li Q, Zeng Y, Ouyang N, et al: Prognostic value of a BCSC-associated microRNA signature in hormone receptor-positive HER2negative breast cancer. EBioMedicine 11: 199-209, 2016.

36. Zheng Q, Cui X, Zhang D, Yang Y, Yan X, Liu M, Niang B, Aziz F, Liu S, Yan Q, et al: miR-200b inhibits proliferation and metastasis of breast cancer by targeting fucosyltransferase IV and $\alpha 1,3$-fucosylated glycans. Oncogenesis 6: e358, 2017.

37. Lee CM, Yang P, Chen LC, Chen CC, Wu SC, Cheng HY and Chang YS: A novel role of RASSF9 in maintaining epidermal homeostasis. PLoS One 6: e17867, 2011. 
38. Ejaz A, Mitterberger MC, Lu Z, Mattesich M, Zwierzina ME, Hörl S, Kaiser A, Viertler HP, Rostek U, Meryk A, et al: Weight loss upregulates the small gtpase diras3 in human white adipose progenitor cells, which negatively regulates adipogenesis and activates autophagy via akt-mtor inhibition. EBioMedicine 6: $149-161,2016$

39. Xu N, Lao Y, Zhang Y and Gillespie DA: AKT: A double-edged sword in cell proliferation and genome stability. J Oncol 2012: 951724, 2012.

40. Zhang J, Tong DD, Xue M, Jiang QY, Wang XF, Yang PB, Ni L, Zhao LY and Huang C: FAM196B acts as oncogene and promotes proliferation of gastric cancer cells through AKT signaling pathway. Cell Mol Biol 63: 18-23, 2017.

41. Maddika S, Ande SR, Wiechec E, Hansen LL, Wesselborg S and Los M: Akt-mediated phosphorylation of CDK2 regulates its dual role in cell cycle progression and apoptosis. J Cell Sci 121: 979-988, 2008.
42. Zhao LY, Zhang J, Guo B, Yang J, Han J, Zhao XG, Wang XF, Liu LY, Li ZF, Song TS, et al: MECP2 promotes cell proliferation by activation ERK1/2 and inhibiting p38 activity in human hepatocellular carcinoma HEPG2 cells. Cell Mol Biol 59: OL1876-OL1881, 2013.

43. Juengel E, Euler S, Maxeiner S, Rutz J, Justin S, Roos F, Khoder W, Nelson K, Bechstein WO and Blaheta RA: Sulforaphane as an adjunctive to everolimus counteracts everolimus resistance in renal cancer cell lines. Phytomedicine 27: 1-7, 2017.

44. Chu X, Zhang T, Wang J, Li M, Zhang X, Tu J, Sun S, Chen X and $\mathrm{Lu} \mathrm{F}$ : Alternative splicing variants of human Fbx4 disturb cyclin D1 proteolysis in human cancer. Biochem Biophys Res Commun 447: 158-164, 2014

45. Li T, Kon N, Jiang L, Tan M, Ludwig T, Zhao Y, Baer R and $\mathrm{Gu}$ W: Tumor suppression in the absence of p53-mediated cell cycle arrest, apoptosis, and senescence. Cell 149: 1269-1283, 2012. 